\title{
Bildbedeutung jenseits der Ikonizität - zur semiotischen Struktur von Paul Klees Tafelbild ,le rouge et le noir (1938)
}

\author{
Felix Thürlemann, Zürich
}

\section{Methodologische Vorbemerkungen}

Es ist in verschiedenen semiotischen Schulen üblich geworden, den Bereich des Bildes (der Malerei, der Photographie, des Films) unter dem Begriff ,ikonische Zeichen" zusammenzufassen, so daß ,ikonisch" synonym zu ,visuell" geworden ist. ${ }^{1}$ Diese Praxis stellt, zumindest für den Bereich der Malerei, an sich schon einen merkwürdigen Anachronismus dar, zu einer Zeit, da ein guter Teil der bildnerischen Produktion sich als afigurativ (ungegenständlich, abstrakt, konstruktiv usw.) versteht. Grundsätzlich problematisch wird diese Praxis jedoch dadurch, daß sich der Begriff des, ikonischen Zeichens" selber für die semiotische Analyse von fragwürdigem Wert erwiesen hat. In der von Morris vulgarisierten Form wird das ,Ikon' innerhalb der fundamentalen Peirce'schen Triade Ikon-Index-Symbol als dasjenige Zeichen bestimmt, das ,dieselben Eigenschaften (properties) " wie der Gegenstand aufweist (Morris 1938, S. 45; vgl. Peirce 1931/35, 2.276 und 2.247). Das Bezugsobjekt wird - in Einengung des Peirce'schen Objekt-Begriffs - üblicherweise als eine Figur der natürlichen Welt verstanden. Wie Umberto Eco (zuletzt 1978, S. 152) aufgezeigt hat, besitzt diese Definition jedoch mehr oder weniger tautologischen Charakter. Das Ikon kann höchstens als ein Zeichen verstanden werden, ,das uns einige Eigenschaften des dargestellten Gegenstandes wiederzugeben scheint" ". Nach Eco funktioniert das ,ikonische Zeichen" meist als Ersatzstimulus (stimulus de remplacement) und weist mit dem „dargestellten Objekt" keine gemeinsamen Eigenschaften auf (Eco 1978, S. 153). In seinem üblichen Gebrauch bezieht sich der Ikon-Begriff demnach auf eine Erfahrung des ,gesunden Menschenverstandes" und bezeichnet das, was in der französischen Semiotik referentielle Illusion (illusion référentielle) genannt wird. ${ }^{3}$ Eine weitere Schwäche einer auf der Peirce'schen Typologie aufbauenden semiotischen Praxis liegt darin, daß sie das Zeichen als ihre Grundeinheit betrachtet. Wie jedoch die Geschichte der modernen Linguistik, vor allem das Beispiel der Phonologie, gezeigt hat, kann das Zeichen nicht als die primäre Einheit eines Sprachsystems angesehen werden. Sowohl die Ausdrucks- als auch die Inhaltsebene (signifiant und signifié) lassen sich in distinktive Einheiten analysieren, die keinen Zeichencharakter mehr haben. ${ }^{4}$

Auf den hier dargelegten methodologischen Grundlagen aufbauend soll nun im folgenden, ausgehend von einem konkreten Analysebeispiel, die Frage gestellt werden nach dem Bedeutungsmodus der afigurativen Malerei besser gesagt: einem möglichen Modus der Bedeutung. Ziel der Analyse ist es darzulegen, daß die Malerei eine Bedeutungsdimension besitzt, die in ihrer 
Existenz nicht von einem Bezug zu den Figuren der Welt abhängt, werde dieser nun als analogisch oder willkürlich aufgefaßt.

,Le rouge et le noir': ein Diskurs über die Farbe

Mit dem Stendhal entliehenen Titel ,le rouge et le noir' (Rot und Schwarz) bezeichnet Paul Klee ein 1938 entstandenes Werk, das sich innerhalb der vielfältigen Produktion des Künstlers durch die Sparsamkeit der angewandten bildnerischen Gestaltungsmittel auszeichnet (Abb.). ${ }^{5}$ Zwei Punkte, ein roter und ein schwarzer, heben sich von einem unregelmäßig gefärbten Grund ab. Figuren und Grund manifestieren dabei zwei fundamental verschiedene chromatische Register: die Figuren haben reine, maximal saturierte und homogen verteilte Farbtöne, der Grund zeigt ein durch verschiedene Sekundärfarben (Rot, Braun, Schwarz) gebrochenes Weiß, das bisweilen die Struktur der Leinwand sichtbar werden läßt; der Anteil der Sekundärfarben ist dabei variabel, aber kontinuierlich graduiert, so daß sich räumliche Sinneffekte einstellen.

Der Titel hat als Zitat einen gewissen ,poetischen“ Beiklang, befindet sich jedoch im übrigen in einem rein denotativen Verhältnis zum Bild, genauer gesagt zu den beiden kreisförmigen Figuren-Elementen, die er in ihrem farbigen Kontrast - rot vs schwarz - bezeichnet. Dieser Kontrast wäre also das, worum es im Bild geht. Wir nehmen nun an, daß jedes visuelle Objekt zwei komplementäre semiotische Dimensionen besitzt, eine chromatische Dimension, welche dem Bereich der „Farben“, und eine eidetische Dimension, welche dem Bereich der „Formen“ im weitesten Sinne (Umriß, Lage, Komposition usw.) entspricht. Unsere These für ,le rouge et le noir" ist somit folgende: wenn die chromatische Dimension Subjekt des Diskurses ist, „das, worum es geht", so stellt die komplementäre, eidetische Dimension das Prädikat dar: die eidetischen Kontraste sollen etwas über das Subjekt, den Farbkontrast, ,aussagen“. ,Le rouge et le noir“ wäre demnach als ein doppelter Diskurs aufzufassen, wobei die eidetische Dimension die Rolle des Explicans, die chromatische Dimension die Rolle des Explicandum spielen würde. Wir nehmen weiterhin an, daß jede der beiden Bilddimensionen, die eidetische und die chromatische, je auf zwei Ebenen analysiert werden kann, auf der Ausdrucks- und der Inhaltsebene. Die eidetischen und chromatischen Qualitäten wären also auf einer primären Lektürestufe in sich schon bedeutungstragend. Schematisch kann die von uns postulierte semiotische Struktur von ,le rouge et le noir' folgendermaßen dargestellt werden:

Explicans eidetische Dimension

eidetischer Ausdruck
Inhalt

Explicandum chromatische Dimension chromatischer Ausdruck [Inhalt]

Es wird zudem postuliert, daß der Inhalt beider Dimensionen identisch ist, wobei der Inhalt der eidetischen Dimension als lesbar, der Inhalt der chromatischen Dimension jedoch als unbekannt erscheint. Es würde somit jene 


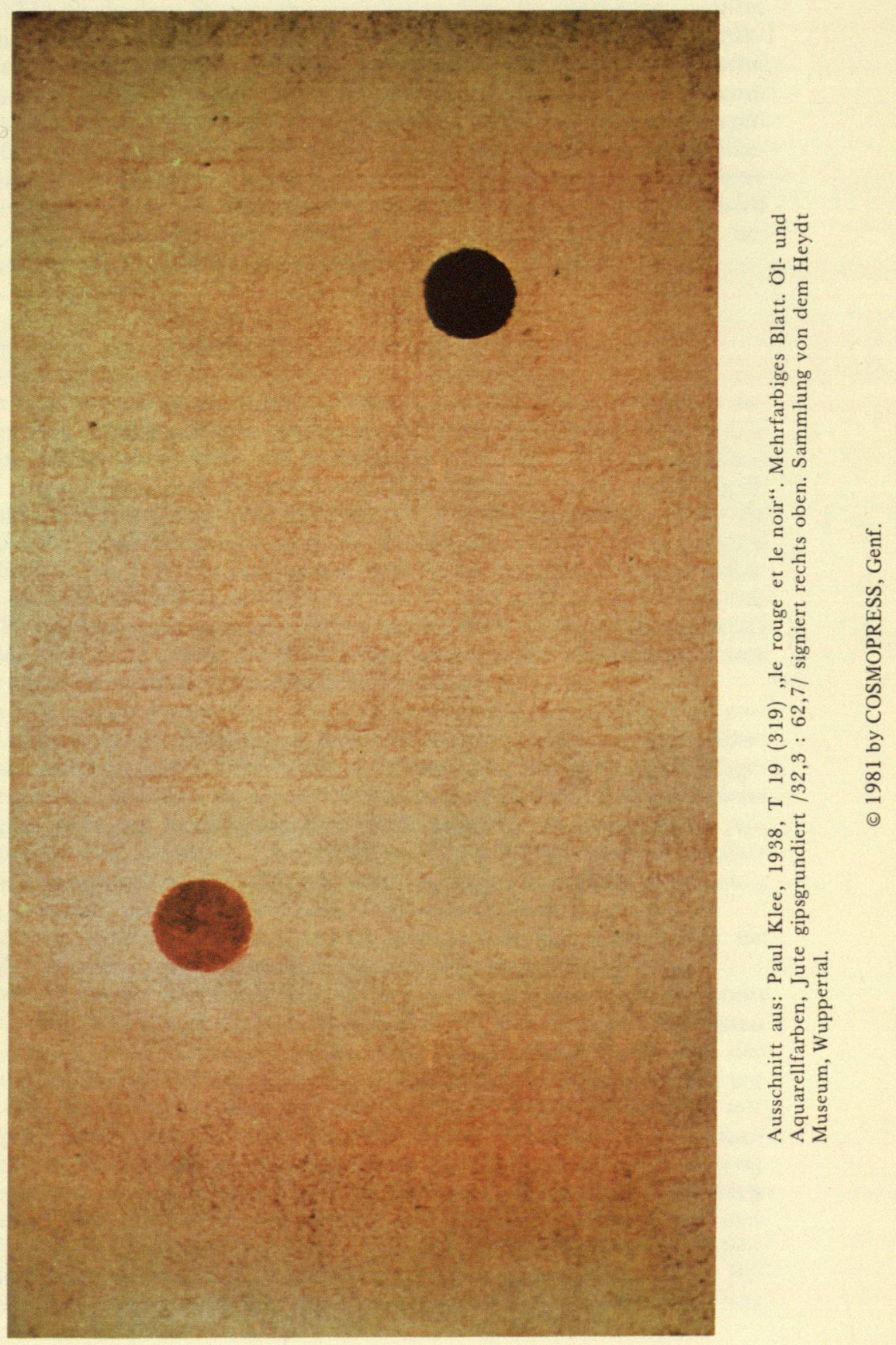


metadiskursive Bedeutungsstruktur vorliegen, die Benveniste (1969, S. 10 und S. 131) einzig dem System der natürlichen Sprache zuerkennen wollte. Ein Indiz dafür, daß wir es tatsächlich mit einer solchen semiotischen Struktur zu tun haben, besteht darin, daß der zu interpretierende chromatische Kontrast von einfacher Natur ist (oder sich zumindest synkretistisch manifestiert), während eine Vielzahl eidetischer Kontraste die Rolle des Explicans erfüllt. Es würde sich somit um die gleiche semiotische Struktur handeln, wie sie im Falle der Definition mittels einer Periphrase vorliegt: eine Ausdruckseinheit einer unbekannten Sprache (hier Sprachdimension) wird mittels Ausdruckseinheiten der gleichen oder einer fremden Sprache bestimmt, deren Inhalt als bekannt vorausgesetzt wird.

\section{Hjelmslevs ,Substanz der kollektiven Wertungen“ neu interpretiert}

Es stellt sich nun die grundsätzliche Frage: von welcher Natur ist dieser Inhalt, der den eidetischen und chromatischen Ausdruckseinheiten auf einer primären Lektürestufe eigen ist? In seinem Artikel von 1954 ,La stratification du langage' hat Louis Hjelmslev ein Modell vorgeschlagen, wonach jede Substanz - des Ausdrucks oder des Inhalts - drei Ebenen enthält, die er die physikalische, soziobiologische und die Ebene der kollektiven Wertungen nennt. So können z. B. die Laute einer Sprache mit den Mitteln der physikalischen Akustik, der artikulatorischen Phonetik oder aber entsprechend den naiven Wertungen einer sozialen Gruppe beschrieben werden. Was die letztgenannte, die Ebene der kollektiven Wertungen betrifft, diejenige, die uns hier vor allem interessiert, bemerkt Hjelmslev (S. 93):

$\mathrm{Da}$ es sich um die Feststellung relativ naiver Wertungen handelt, deren einziges ,theoretisches' Fundament darin zu suchen ist, was wir Verhaltensnormen genannt haben, die in die Auffassungen der Gesellschaft eingegangen sind, scheint es so, als ob die ,Metasprache', deren sich eine solche Disziplin bedienen könnte, um ihre Ziele zu erreichen, lediglich die Alltagssprache sein könnte. [...] wir geben einige zufällig ausgewählte, aber relativ wahrscheinliche Beispiele wie ,hell": ,,dunkel“", ,stark“" ,schwach“, ,lang": ,kurz", ,hoch": ,tief", ,schwer": ,leicht" usw.

Hjelmslev fügt schließlich hinzu, daß zu den notwendigen Vorarbeiten für eine solche Beschreibung das Studium der Synästhesie gehöre.

Obwohl Hjelmslev seine Beispiele in der Ausdruckssubstanz der natürlichen Sprachen wählt, postuliert er, daß es sich mit allen übrigen Substanzen gleich verhalte. Eine Ebene der kollektiven Wertungen ist im Bereich der chromatischen Substanz tatsächlich leicht auszumachen. Es handelt sich um den Modus der Farbwahmehmung, den Goethe in seiner Farbenlehre mit dem Begriff ,sinnlich-sit tliche Wirkung der Farbe“ bezeichnet hat (didaktischer Teil, sechste Abteilung). So besitzt nach Goethe das Gelb ,eine heitere, muntere, sanft reizende Eigenschaft" $(\$ 766)$, und ,Zimmer, die rein blau austapeziert sind, erscheinen gewissermaßen weit, aber eigentlich leer und kalt" (§ 783). Wir schlagen nun vor, Hjelmslev ,kollektive Wertung“ nicht mehr als Modus der Substanzbeschreibung zu betrachten, sondern als Beschreibung des Inhalts, und zwar immer dann, wenn sie für die Lektüre eines 
Prozesses relevant ist, was für ,le rouge et le noir von Klee der Fall zu sein scheint.

Die bisher wohl besten Beschreibungen der Farben auf der Ebene der kollektiven Wertungen (sinnlich-sittliche Dimension) hat Kandinsky 1912 in der Schrift Ueber das Geistige in der Kunst gegeben. Wir möchten nun zwei Auszüge aus seinen Beschreibungen der Farben Rot und Schwarz anführen, und zwar im Sinne einer Hypothese in Bezug auf die Bedeutung unseres Analyseobjektes. Wenn nämlich unser Modell von der semiotischen Struktur von ,le rouge et le noir' richtig ist, so müßte die wertende Lektüre der eidetischen Kontraste, die wir im letzten Kapitel vornehmen werden, derjenigen des Farbkontrastes rot vs schwarz entsprechen. Kandinsky schreibt (S. 99):

Das Rot, so wie man es sich denkt, als grenzenlose, charakteristisch warme Farbe, wirkt innerlich als eine sehr lebendige, lebhafte, unruhige Farbe [...] Das helle warme Rot (Satum) [diese Benennung entspricht gut dem in ,le rouge et le noir" verwendeten Farbton] hat eine gewisse Ahnlichkeit mit Mittelgelb (enthält auch als Pigment ziemlich viel Gelb) und erweckt das GefühI von Kraft, Energie, Streben, Entschlossenheit, Freude, Triumph (lauter) usw. Es erinnert musikalisch auch an den Klang der Fanfaren, wobei die Tuba beiklingt - hartnäckiger, aufdringlicher, starker Ton.

Und zum Schwarz (S. 98):

Und wie ein Nichts ohne Möglichkeit, wie ein totes Nichts nach dem Erlöschen der Sonne, wie ein ewiges Schweigen ohne Zukunft und Hoffnung klingt innerlich das Schwarz. Es ist musikalisch dargestellt wie eine vollständig abschließende Pause, nach welcher eine Fortsetzung kommt wie der Beginn einer andern Welt, da das durch diese Pause Abgeschlossene für alle Zeiten beendigt, ausgebildet ist: der Kreis ist geschlossen. Das Schwarz ist etwas Erloschenes, wie ein ausgebrannter Scheiterhaufen, etwas Unbewegliches, wie eine Leiche, was $\mathrm{zu}$ allen Ereignissen nicht fühlend steht und alles von sich gleiten läßt. Es ist wie das Schweigen des Körpers nach dem Tode, dem Abschluß des Lebens. Das ist äußerlich die klangloseste Farbe, auf welcher deswegen jede andere Farbe, auch die am schwächsten klingende, stärker und präziser klingt.

Wir können feststellen, daß in der Beschreibung von Schwarz ein Großteil der wertenden Ausdrücke, die das helle Rot charakterisieren, in negierter Form wieder vorkommen. In vielerlei Hinsicht kann somit Schwarz als antonyme Farbe zum hellen Rot verstanden werden. Die nachfolgende Liste faßt die wichtigsten Charakteristika der beiden Farbtöne nach Kandinsky schematisch zusammen.

Rot

Schwarz

warm

lebhaft, lebendig

Kraft, Energie

Streben, grenzenlos

Freude, Triumph

hartnäckiger, aufdringlicher, starker Ton

unbeweglich, tot

ohne Möglichkeit, abgeschlossen

Schweigen, klanglos 
Kandinsky hat seine Überlegungen zur ,,psychischen Wirkung“" der elementaren visuellen Gestaltungsmittel nicht nur auf die Farbe beschränkt. „Die Form selbst, wenn sie auch ganz abstrakt ist und einer geometrischen gleicht, hat ihren innern Klang, ist ein geistiges Wesen mit Eigenschaften, dic mit dieser Form identisch sind“ (S. 68). Er bemerkt dann (S. 68f), ,daß manche Farbe durch manche Form in ihrem Wert unterstrichen wird und durch andere abgestumpft. Jedenfalls spitze Farben klingen in ihrer Eigenschaft stärker in spitzer Form (z. B. Gelb im Dreieck). Die zur Vertiefung geneigten werden in dieser Wirkung durch runde Formen erhöht (z. B. Blau im Kreis). “ Damit ist die These von der Homologierbarkeit von eidetischen und chromatischen Qualitäten ausgesprochen, welche auch die Grundlage für unsere $\mathrm{Hy}$ pothese zur semiotischen Struktur von ,le rouge et le noir' bildet. Daß diese Homologierbarkeit auf einem gemeinsamen Inhalt von wertender Natur beruht, geht aus den Zitaten Kandinskys ebenfalls hervor. ${ }^{6}$ Während Kandinsky sich in seinen Schriften immer wieder mit dem Verhältnis zwischen Form und Farbe beschäftigt hat, so scheinen diesbezügliche Außerungen in den theoretischen Schriften Klees, jedenfalls in den bis anhin veröffentlichten, fast ganz zu fehlen. Es ist mir nur ein Satz aus der Düsseldorfer Zeit (1931-33) bekannt, wo Klee seine Besprechung einer Schülerarbeit folgendermaßen zusammenfaßt (Petitpierre 1957, S. 41): ,... das wäre in gewissem Sinne eine Ubereinstimmung von Form und Farbe. “7

\section{Wertung der eidetischen Kontraste: die Bedeutung}

,Le rouge et le noir' manifestiert eidetische Kontraste verschiedenster Kategorien, solche des Umrisses, der Lage und der Einordnung in das Achsenschema des Formats. Hinzu kommen die Kontraste in der chromatischen Gestaltung des Hintergrundes, die über ihre räumlichen Sinneffekte mit den eidetischen Kontrasten zusammen betrachtet werden können. Das Bild besitzt ein Querformat, dessen Seitenlängen annähernd im Verhältnis 2:1 stehen, was zur Folge hat, daß sich die Bildfläche einer Unterteilung in zwei Quadrate anbietet. Wir können somit eine linke Seite der Grundfläche mit dem ihm zugeordneten roten Element (beide zusammen werden im folgenden mit $\mathrm{R}$ bezeichnet) und eine rechte Seite mit dem schwarzen Element (S) unterscheiden. Diese beiden Seiten und die mit ihnen metonymisch verbundenen Elemente werden nun in einer rein syntagmatischen Lektüre einander kontrastiv gegenübergestellt; $d . h$. wir verzichten auf eine Temporalisierung der Lektüre durch die Wahl einer Lektürerichtung. Es gibt keine Indizien, die eine derartige Lektüre für ,le rouge et le noir' nahelegen würden. In der nachfolgenden Liste sind die eidetischen Kontraste, nach Kategorien geordnet, aufgeführt.

\section{Umriß}

$\mathrm{R}$ Kreis in der Vertikalen leicht gelängt

S Kreis in der Vertikalen leicht abgeflacht (oberer Rand) 
Lage

$\mathrm{R}$ links

$\mathrm{R}$ oben

$S$ rechts

$S$ unten

Einordnung

$\mathrm{R}$ unregelmäßige Distanz zu den

beiden Seiten (links und oben)

R Stellung rechts von der Mittel-

vertikalen des linken Vierecks, exzentrisch

$\mathrm{R}$ exzentrische Stellung zur

Mittelhorizontalen

chromatische Hintergrundsgestaltung

$\mathrm{R}$ veränderlicher Helligkeitswert

des Grundes, um das Element auf-

S Distanz zu den beiden Seiten (rechts und unten) ungefähr gleich, regelmäßig

S Stellung auf der Mittelvertikalen des rechten Vierecks, nicht exzentrisch

S nicht exzentrische Stellung zur Mittelhorizontalen

gehellt; vertikaler Streifen

S homogener Helligkeitswert

Als erstes fällt auf, daß ein Großteil der untersuchten Kontraste, jene der Einordnung und der chromatischen Hintergrundgestaltung, eine fast identische Charakterisierung aufweisen. Die Seite R erscheint als variiert (unregelmäßig, exzentrisch, veränderlich), während sich die Seite $\mathrm{S}$ als einförmig (regelmäßig, nicht exzentrisch, homogen) charakterisieren läßt. Diese Adjektive enthalten in sich schon Elemente der Wertung, die wir mit den Begriffen ,dynamisch" vs ,"statisch“" verdeutlichen wollen. ${ }^{8}$ Wir gelangen so zu einer ersten wertenden Umsetzung eidetischer Kontraste in Form eines doppelten Homologationsschemas:

R : S : : variiert : einförmig : :,dynamisch“ : ,,statisch“

Was die Kategorie der Lage betrifft, scheint eine Wertung des horizontalen Kontrastes links vs rechts außerhalb einer temporalisierten Lektüre nicht möglich. Für den vertikalen Kontrast schlagen wir die übliche Wertung vor:

R : S :: oben : unten :: ,leicht“ : „schwer“

Schwieriger scheint eine direkte Umsetzung des Umrißkontrastes gelängt vs abgeflacht (in Bezug zur Vertikalen) zu sein. Wir haben festgestellt, daß ebenfalls bei der Hintergrundsgestaltung in $\mathrm{R}$ die Vertikale markiert ist. Diese beiden Kategorien zusammen ergeben einen Sinneffekt ${ }^{9}$, den man als eine genauere Bestimmung des Wertes „dynamisch“ verstehen kann. Das rote Element erscheint als eine Figur, die der Bewegung fähig ist, und zwar vornehmlich in vertikaler Richtung, genauer von unten nach oben, da man die (nach oben) exzentrische Lage des roten Elementes als Ergebnis einer , aufsteigenden" Bewegung auffassen kann. Weniger betont, aber ebenfalls vorhanden sind Sinneffekte der Bewegung in anderen Richtungen. Das rote Element scheint, wegen der Aufhellung des Hintergrundes, rundum ,auszustrahlen“" Während die Verdunklung auf der rechten Seite (S) den Effekt materieller Präsenz ergibt, eine Fläche simulierend, an der das schwarze Element gleich- 
sam ,angeheftet" ist, so ergibt die Aufhellung auf der linken Seite (R) den Effekt der Leere; das rote Element scheint im Raum zu schweben und hat die Tendenz, sich auf einer imaginären Tiefenachse auf den Betrachter hin zu bewegen.

Zusammengefaßt ist $\mathrm{R}$, und damit die rote Farbe, als ,dynamisch, leicht und nach allen Seiten, vor allem aber nach oben hin sich bewegend" charakterisiert, wohingegen für $\mathrm{S}$, und damit für die schwarze Farbe, alle diese Wertungen in negierter Form erscheinen. Dieses Resultat der Lektüre der eidetischen Kontraste auf der Ebene der kollektiven Wertungen entspricht in wesentlichen Teilen den Charakteristiken, welche wir aus Kandinskys Beschreibungen von Rot und Schwarz entnommen haben (vgl. das kontrastierende Schema S. 7). Damit ist unsere Hypothese bestätigt, wonach der Inhalt der eidetischen Kontraste in ,le rouge et le noir" als metasprachliche Bestimmung des Inhalts des chromatischen Kontrastes rot vs schwarz anzusehen ist. ${ }^{10}$

,Le rouge et le noir" erscheint uns als eine Reflexion über die Möglichkeit und die Natur der Bedeutung in der afigurativen Malerei. Die afigurative Malerei, in all ihren Variationen, setzt die Existenz einer nicht ikonischen Bedeutung voraus, einer Bedeutung, die nicht an die Figuren der Welt gebunden ist. Kandinsky hat in seinen theoretischen Schriften immer wieder versucht, diese den bildnerischen Gestaltungsmitteln immanente Bedeutungsdimension aufzuzeigen. Diesen Rechtfertigungsversuch einer rein visuellen Sprache unternahm er jedoch mit Hilfe eines anderen Sprachsystems, dem der natürichen Sprache. In ,le rouge et le noir' hat Klee dazu einen unterschiedlichen, dem Problem einzig adäquaten Weg gewählt, er überließ es der Malerei selber, ihre semiotische Selbständigkeit zu beweisen. ${ }^{11}$

\section{Anmerkungen}

1 So z. B. bei Cresti 1972, wo ,iconique' dem Begriff ,verbal' gegenübergestellt wird (vgl. etwa Anm. 8, S. 47). Es muß jedoch festgestellt werden, daß Cresti in ihrer interessanten Analyse die Definition des ,ikonischen' Zeichens als analoges Zeichen in Frage stellt.

2 Eco 1972, S. 213 (Hervorhebung von mir).

${ }^{3}$ Peirce selber hat dieses Problem gesehen. Zum Portrait einer dem Betrachter unbekannten Person bemerkt er (Peirce 1931/35, 2.92): , in fact, it is not a pure Icon, because $I$ am greatly influenced by knowing that it is an effect, through the artist, caused by the original's appearance, and is thus in a genuine Obsistent relation to that original. Besides, I know that portraits have but the slightest resemblance to their originals, except in certain conventional respects, and after a conventional scale of values, etc."

4 Unsere Kritik des Begriffs des, ikonischen Zeichens' schließt sich an diejenige von A. J. Greimas an; vgl. Greimas-Courtés 1979 unter, iconicité ${ }^{\prime}(1)^{`}:$, ,Si l'on considère [...] que la définition du signe par ce qu'il n'est pas est sćmiotiquement non pertinente et que, d'autre part, la sémiotique ne devient opératoire que lorsqu'elle situe ses analyses en-deçà ou au-delà du signe, la classification proposée [par Peirce], sans être gênante, n'offre que peu d'intérêt."

5 Am 2. Semiotischen Kolloquium in Regensburg wurden die Thesen an einem anderen Werk Paul Klees, ,Pflanzen-Analytisches* (1932/229), erläutert. Der beschränkte Raum, der im Rahmen dieser Publikation zur Verfügung steht, ließ es geraten erscheinen, ein anderes Analyseobjekt zu wählen. 
,Le rouge et le noir' wurde von Klee unter seine Tafelbilder eingeordnet. Es trägt die Verweisnummer auf das Werkverzeichnis 1938/T 19. Die Technik ist Oel und Kleisterfarbe auf gipsgrundierter Jute, das Format $32,5 \times 63 \mathrm{~cm}$. Ehemals in der Sammlung von Prof. Anselmino befindet sich das Werk heute im Von der Heydt-Museum der Stadt Wuppertal. Farbige Reproduktion in: Klee 1971, S. 14, und Aust 1977b, S. 223.

${ }^{6}$ Es ist interessant zu sehen, daß Max Lüscher seinen bekannten Farbtest neuerdings durch einen Formtest ergänzt hat, welche beide zusammen angewandt werden. Dieses Vorgehen setzt ebenfalls voraus, daß bestimmte Farbqualitäten und Formqualitäten die gleiche primäre Bedeutung besitzen.

7 Die im Heft 9/48 (113-115) von Paul Klees pädagogischem Nachlaß zu den einzelnen Farben angegebenen Formen haben modellhaften, erklärenden Charakter im Zusammenhang mit den Aufhellungs- und Verdunkelungsmöglichkeiten der jeweiligen Farben. Daß sich die Schemata (u. a. Dreieck für Gelb, Quadrat für Rot, Ellipse für Blau) dem von Kandinsky aufgestellten System nähern, ist an sich zufällig; der Zufall ist jedoch von Klee, der die Nähe zu ,Meister Kandinsky“ hier ausdrücklich vermerkt, wahrscheinlich etwas gesteuert worden. (Den Hinweis auf diese noch unveröffentlichte Stelle aus dem Nachlaß verdanke ich Dr. Christian Geelhaar, Konservator am Kunstmuseum Basel.)

8 Diese gegensätzliche Wertung könnte auch mit den mehr symbolischen Begriffen „lebendig“"vs "tot" wiedergegeben werden. So könnte man etwa im Bild, le rouge et le noir" eine Visualisierung der Unvereinbarkeit der beiden konträren Prinzipien ,Leben“ und ,"Tod" sehen (so Aust, 1977a). Dazu soll jedoch bemerkt werden, daß es uns nicht in erster Linie um eine interpretative Lektüre des Bildes von Klee geht, die notwendigerweise reduktiven Charakter hätte, insofern sie nur eine mögliche Applikation darstellen würde. Uns geht es vielmehr darum, an einem Beispiel den komplexen Proze $\beta$ der Bedeutungskonstituierung zu analysieren, als Beitrag zu einer formellen Semiotik im Sinne von A. J. Greimas (1970, S. 16): ,une sémiotique formelle qui ne chercherait à rendre compte que des articulations et des manipulations des contenus quelconques."

9 Der Begriff "Sinneffekt" wird hier entsprechend der Definition von A. J. Greimas (1978, S. 2) gebraucht: ,on entend par effet de sens un sémantisme confus, tel qu'il est susceptible d'être saisi de manière syncrétique à un niveau quelconque du parcours de la production du sens."

10 Wenn wir das Verhältnis der eidetischen Kontraste zu diesem internen Referenzobjekt (dem chromatischen Kontrast) mit den Begriffen der Peirce'schen Fundamentaltriade charakterisieren wollten, so müßte man überraschenderweise von einem ikonischen Bezug sprechen. Bei den Eigenschaften, die dem ,Zeichen (eidetische Dimension) mit dem ,Objekt' (chromatische Dimension) gemeinsam wären, würde es sich um die gemeinsamen Bedeutungselemente handeln. Es braucht aber wohl nicht besonders her vorgehoben werden, $\mathrm{daB}$ eine solche Interpretation gegen den Geist der Peirce'schen Semiotik gerichtet ist, da es sich bei ihr - um in den Begriffen Hjelmslevs zu sprechen - um eine transzendentale Sprachtheorie handelt.

Nach Abschluß der Analyse bin ich auf einen Text gestoßen, der zweifellos als Ausgangspunkt für Klees Tafelbild betrachtet werden muß. Es handelt sich um einen Aufsatz von Kandinsky - daß es gerade dieser Autor ist, überrascht uns nicht mehr den er 1935, also drei Jahre vor ,le rouge et le noir', in Les Cahiers d'Art 5/6 veröffentlicht hat. Unter dem Titel „Toile vide, etc.“ beschreibt Kandinsky dort den Inhalt des schwarzen Kreises und des roten Kreises in zwei korrelierten Abschnitten (S. 117):

Cercle noir - tonnerre éloigné, un monde pour soi qui ne semble ne se soucier de rien, un se-retirer-en-soi-même, une conclusion sur place. Un ,me voilà “ dit lentement et un peu froidement.

Cercle rouge - tient ferme, garde sa place, approfondi en soi-même. Mais en même temps il chemine puisqu'il voudrait avoir toutes les autres places pour soi, - ainsi il rayonne au-dessus de tout obstacle jusqu'au coin le plus éloigné. Eclair et tonnerre en même temps. Un ,me voilà " passionné.

Kandinskys Text ist original in französischer Sprache verfaßt, was die Verwendung von Stendhals Roman-Titel durch Klee zusätzlich erklärt. Ein Vergleich unserer Inhalts- 
analyse von ,le rouge et le noir" mit dem Inhalt der metasprachlichen Bestimmung der beiden Farb-Form-Komplexe (,,cercle noir", „,cercle rouge") durch Kandinsky läßt auf weitgehende Ubereinstimmung schließen.

\section{Literatur}

Aust, Günter, 1977a: Paul Klee - le rouge et le noir, in: Jahresbericht des Von der HeydtMuseums 1977.

Aust, Günter, 1977b: Das Von der Heydt-Museum in Wuppertal, Recklinghausen.

Benveniste, Emile, 1969: Sémiologie de la langue, in: Semiotica 1, 1969, S. $1-12$ u. $127-135$.

Cresti, Emanuela, 1972: Oppositions iconiques dans une image de bande dessinée reproduite par Lichtenstein, in: VS 2/4, 1972, S. 41-62.

Eco, Umberto, 1972: Einführung in die Semiotik, München.

Eco, Umberto, 1978: Pour une reformulation du concept de signe iconique, in: Communications 29, 1978, S. 141-191.

Goethe, Johann Wolfgang, 1810: Farbenlehre, zit. nach: Naturwissenschaftliche Schrif. ten 1. Teil, Artemis-Gedenkausgabe Bd. 16, Zürich/Stuttgart 1964.

Greimas, Algirdas Julien, 1970: Du sens - Essais sémiotiques, Paris.

Greimas, Algirdas Julien, 1978: Pour une sémiotique des passions, in: Le Bulletin (organe du groupe de recherches sémio-linguistiques, E.H.E.S.S.) 6, 1978, S. $1-4$.

Greimas, Algirdas Julien/Courtés, Joseph, 1979: Sémiotique - Dictionnaire raisonné de la théorie du langage, Paris.

Hjelmslev, Louis, 1954: La stratification du langage, in: Word 10, 1954, S. 36-68; zit. nach der deutschen Ubers. in L. H.: Aufsätze zur Sprach wissenschaft, Stuttgart, 1974.

Kandinsky, Wassily, 1912: Uber das Geistige in der Kunst, zit. nach der 4. Aufl., Bern, 1952.

Klee, Paul, 1971 : Das bildnerische Denken - Form-und Gestaltungslehre, Bd. 1, 3. Aufl., Basel/Stuttgart.

Morris, Charles W., 1938: Foundations of the Theory of Signs, Chicago; deutsch in C. W. Morris: Grundlagen der Zeichentheorie - Asthetik und Zeichentheorie, München ${ }^{2} 1975$, S. $15-88$.

Peirce, Charles S., 1931/35: Collected Papers, Cambridge Mass.

Petitpierre, Petra, 1957: Aus der Malklasse von Paul Klee, Bern. 